\title{
Experimental probes of traversable wormholes
}

\author{
Dongsu Bak, ${ }^{a, c}$ Chanju $\mathrm{Kim}^{b}$ and Sang-Heon $\mathbf{Y i}^{a}$ \\ ${ }^{a}$ Physics Department, University of Seoul, \\ Seoul 02504, Korea \\ ${ }^{b}$ Department of Physics, Ewha Womans University, \\ Seoul 03760, Korea \\ ${ }^{c}$ Natural Science Research Institute, University of Seoul, \\ Seoul 02504, Korea \\ E-mail: dsbak@uos.ac.kr, cjkim@ewha.ac.kr, shyi704@uos.ac.kr
}

ABSTRACT: We propose possible probes which could be used to demonstrate experimentally the existence of the bulk and the formation of a traversable wormhole purely in terms of boundary operations only. In the two-dimensional Einstein-dilaton gravity, the traversable wormhole is realized by turning on a double trace interaction which couples the two boundaries of the $\mathrm{AdS}_{2}$ black hole. Signals can propagate in the traversable wormhole through two different channels. The boundary channel is direct and instantaneous, while the bulk channel respects the bulk causality and takes a certain amount of time to complete signaling. In the latter case, we show that the signal frequency detected on the other side is highly modulated in general. The time delay as well as the frequency-modulation pattern could then be clear indications that the signal comes out through the bulk channel. We discuss the characteristics of the observed signal more explicitly for simple transitional configurations of the black hole from/to the eternal traversable wormhole.

KeYwords: 2D Gravity, AdS-CFT Correspondence, Black Holes

ARXiv EPrint: 1907.13465 


\section{Contents}

1 Introduction 1

2 Bulk description of traversable wormholes 2

$\begin{array}{llr}3 & \text { Signaling in traversable wormhole } & 6\end{array}$

4 Experimental probes of wormholes $\quad 9$

5 Conclusions 12

$\begin{array}{ll}\text { A Perturbative solution to ETW } & 13\end{array}$

B $\Delta=\frac{1}{2}$ case $\quad 14$

\section{Introduction}

Recently, there are remarkable developments on two-dimensional Einstein-dilaton gravity, dubbed as the Jackiw-Teitelboim (JT) model $[1,2]$. The absence of the bulk gravitational degree of freedom in this model lowers the computational and conceptual hurdles and the resultant boundary theory, which is known as the Schwarzian theory [3-6], has led to full quantum computations, very recently [7-9] (see [10] in some other direction). In the context of the AdS/CFT correspondence, the boundary dual theory is also identified with the Sachdev-Ye-Kitaev (SYK) model [11], which spurs various works on expanding our understanding. These developments give us various new insights in quantum gravity, though those seem to be strongly model-dependent at this stage. However, since the JT model appears as a universal low energy limit of the near horizon on extremal black holes in the form of nearly $\mathrm{AdS}_{2}$ or nearly $\mathrm{dS}_{2}$ gravity, it would give us useful insights to the quantum nature of black holes and even in the quantum nature of cosmology $[12,13]$.

In this note we shall be mainly concerned with bulk aspects of traversable wormholes in the JT model, which requires the violation of the averaged null energy condition (ANEC) in a specific way $[6,14,15]$. Although this specific ANEC violating left/right $(\mathrm{L} / \mathrm{R})$ boundary interaction or boundary sources are non-local and lead to a direct instantaneous interaction between $\mathrm{L} / \mathrm{R}$ boundaries, the bulk locality and causality seem to be preserved in some sense. The usual bulk gravitational interpretation of traversable wormholes in our model is completely consistent and provides a powerful tool for the probe to the GR=QM picture [16-18]. Since the bulk causality seems crucial in this context in order to take the existence of the bulk as a reality, it is very illuminating to see clearly the bulk causality features in our model. Specifically, we shall be interested in sending signals from one boundary to the other one for black holes/wormholes transition configuration, which would be a characteristic experiment to see the bulk causality. 
This note is organized as follows. In section 2, we provide a brief summary of our setup and recall our bulk solution to the black holes/wormholes transition. In section 3 , we describe how to send the signals from one boundary to the other one through the bulk and what the bulk causality tells us in this process. In section 4, we suggest how to probe the bulk by a certain experiment for two coupled boundary systems and what is the specific feature of this experiment, implying the bulk as a reality, compared to the usual direct interaction between them. In the final section, we conclude with some comments.

\section{Bulk description of traversable wormholes}

In this section we summarize our bulk construction of black hole/wormhole transition in $[19,20]$. Our setup is based on two-dimensional Einstein-dilaton gravity, known as the JT model. It consists of a dilaton field $\phi$, a metric $g$, and a free massive scalar field $\chi$ which does not couple directly the dilaton field. Explicitly, its action is given by

$$
I=I_{\text {top }}+\frac{1}{16 \pi G} \int_{M} d^{2} x \sqrt{-g} \phi\left(R+\frac{2}{\ell^{2}}\right)+I_{\text {surf }}+I_{M}(g, \chi),
$$

where

$$
\begin{aligned}
I_{\mathrm{top}} & =\frac{\phi_{0}}{16 \pi G} \int_{M} d^{2} x \sqrt{-g} R, \quad I_{\text {surf }}=-\frac{1}{8 \pi G} \int_{\partial M} \sqrt{-\gamma}\left(\phi_{0}+\phi\right) K, \\
I_{M} & =-\frac{1}{2} \int_{M} d^{2} x \sqrt{-g}\left(\nabla \chi \cdot \nabla \chi+m^{2} \chi^{2}\right) .
\end{aligned}
$$

Here, $\ell$ is the AdS radius, and $\gamma_{i j}$ and $K$ denote the induced metric and the extrinsic curvature at $\partial M$, respectively. The dilaton field $\phi$ plays the role of the Lagrange multiplier and sets the metric to be $\mathrm{AdS}_{2}$ which can be written in the global coordinates as

$$
d s^{2}=\frac{\ell^{2}}{\cos ^{2} \mu}\left(-d \tau^{2}+d \mu^{2}\right), \quad \mu \in\left[-\frac{\pi}{2}, \frac{\pi}{2}\right] .
$$

The metric variation leads to the equations of motion for the dilaton field $\phi$ as

$$
\nabla_{a} \nabla_{b} \phi-g_{a b} \nabla^{2} \phi+g_{a b} \phi=-8 \pi G T_{a b}
$$

where $T_{a b}$ is the stress tensor of the scalar field $\chi$,

$$
T_{a b}=\nabla_{a} \chi \nabla_{b} \chi-\frac{1}{2} g_{a b}\left(\nabla \chi \cdot \nabla \chi+m^{2} \chi^{2}\right)
$$

In this note, we shall set $8 \pi G=1$ for the simplicity. With $T_{a b}=0$, the non-vanishing general vacuum solution for the dilaton field, which could be interpreted as black holes, can be obtained in the form of

$$
\phi_{\mathrm{BH}}=\bar{\phi} L \frac{\cos \tau}{\cos \mu},
$$

where we used $\mathrm{SL}(2, \mathbf{R})$ to set the solution in the above form [19]. Here, the length dimension parameter $L$ could be interpreted as the horizon radius, which can be shown as 
follows: by an appropriate coordinate transformation the above $\mathrm{AdS}_{2}$ metric can be set in the form of the black hole metric as

$$
d s^{2}=-\frac{r^{2}-L^{2}}{\ell^{2}} d t^{2}+\frac{\ell^{2}}{r^{2}-L^{2}} d r^{2} .
$$

We are in the low energy regime when $L \ll \ell$.

In the context of nearly $\mathrm{AdS}_{2}$ gravity [5, 21], the degrees of freedom in this system reside on the boundaries and their dynamics may be described by a Schwarzian theory. In this description, boundary values of the metric and the dilaton could be taken in $\epsilon \rightarrow 0$ limit as

$$
\left.d s^{2}\right|_{\partial M}=-\frac{1}{\epsilon^{2}} d \tilde{u}^{2},\left.\quad \phi\right|_{\partial M}=\frac{\ell \bar{\phi}}{\epsilon},
$$

where $\tilde{u}$ denotes the (proper) boundary time. In order to construct the traversable wormholes in the bulk [14], we need the non-trivial scalar field $\chi$, whose boundary behavior is given by

$$
\left.\chi\right|_{\partial M}=\epsilon^{\Delta} \tilde{\alpha}+\cdots+\epsilon^{1-\Delta} \tilde{\beta}+\cdots,
$$

and the mixed boundary condition corresponds to

$$
\tilde{\beta}_{L / R} \propto \tilde{\alpha}_{R / L},
$$

where the subscript $L / R$ refers to the left/right boundary, respectively. According to the AdS/CFT correspondence, this boundary condition corresponds to the double trace deformation of the boundary theory [22, 23], and may be written explicitly as the deformation of the boundary Hamiltonian

$$
\delta H(\tilde{u})=-h(\tilde{u}) \mathcal{O}_{R}(\tilde{u}) \mathcal{O}_{L}(\tilde{u})
$$

where $\mathcal{O}_{R, L}$ are scalar operators of dimension $\Delta \in(0,1 / 2)$, dual to $\chi$. On the other hand, by taking $\tilde{\beta}$ as the source term, this deformation could be realized as the non-local interaction with the $\mathrm{SL}(2, \mathbf{R})$ gauge constraint (see $[5,21,24])$. In this note, we take the $\mathrm{L} / \mathrm{R}$ boundary proper time as the same one $\tilde{u}$. One may equivalently describe the system by the Schwarzian derivatives at the boundaries with an interaction term [6, 25],

$$
S=\int d \tilde{u}\left[-\phi_{L} \operatorname{Sch}(\tilde{u})-\phi_{R} \operatorname{Sch}(\tilde{u})\right]+S_{\mathrm{int}}
$$

where

$$
\operatorname{Sch}(\tilde{u}) \equiv\left\{\tan \frac{\tau(\tilde{u})}{2}, \tilde{u}\right\}, \quad S_{\mathrm{int}} \equiv \frac{g}{2^{2 \Delta}} \int d \tilde{u}\left[\frac{\tau_{L}^{\prime}(\tilde{u}) \tau_{R}^{\prime}(\tilde{u})}{\cos ^{2} \frac{\tau_{L}(\tilde{u})-\tau_{R}(\tilde{u})}{2}}\right]^{\Delta} .
$$

Here, $\phi_{L}=\phi_{R}$ can be identified with $\bar{\phi}$ in the bulk and $g$ is a coupling proportional to the parameter $h$, whose explicit identification is given by [19]

$$
g=\frac{h}{2 \pi} \frac{2^{2 \Delta-1} \Gamma^{2}(\Delta)}{\Gamma(2 \Delta)}
$$


It has been shown $[5,24]$ that one may need to impose the appropriate gauge choice for the ghost-free dynamics in this Schwarzian description.

Though the metric is not affected by the presence of the matter field $\chi$, the deformation (2.11) via the scalar field $\chi$ affect the dilaton field $\phi$ through (2.4). The general solution of (2.4) can be written as [19]

$$
\phi=\phi_{\mathrm{hom}}+\varphi
$$

where $\phi_{\text {hom }}$ denotes a vacuum solution (i.e. $T_{a b}=0$ case) and $\varphi$ is given by

$$
\varphi(u, v)=\int_{u_{0}}^{u} d p \frac{\sin (p-u) \cos (p-v)}{\cos (u-v)} T_{u u}(p, v) .
$$

Here, $u$ and $v$ are global null coordinates defined by $u \equiv \frac{1}{2}(\tau+\mu), v \equiv \frac{1}{2}(\tau-\mu)$.

Now, we consider eternal traversable wormholes (ETWs) in this setup. As was shown in [20], by turning on the double trace interaction from the infinite past with the symmetric choice $\tau_{L}(\tilde{u})=\tau_{R}(\tilde{u})=\tau(\tilde{u})$ and by taking $\phi_{\text {hom }}=0$, the ETWs could be realized by the dilaton field solution as

$$
\phi_{\mathrm{ETW}}(u, v)=\frac{1}{2} \varphi_{\mathbf{I}}(u, v),
$$

where

$$
\begin{aligned}
\varphi_{\mathbf{I}}(u, v)= & \frac{4 \ell^{2} \bar{h} \Delta N_{\Delta}}{2^{2 \Delta}} \frac{B(2-\Delta, 2-\Delta)}{1-\Delta} \frac{\sin ^{3-2 \Delta}|u-v|}{\cos |u-v|} \\
& \times F\left(1-\Delta, 1-\Delta ; \frac{5}{2}-\Delta \mid \sin ^{2}(u-v)\right) .
\end{aligned}
$$

Here, we would like to remind that the final expression of the dilaton field $\phi$ is obtained by a certain averaging procedure from a subregion contribution denoted as $\varphi_{\mathbf{I}}$. In this case, the sources at L/R boundaries are infinitely spread, and so one needs to add the subregion contribution appropriately. See [20] for the details and the notation. Whereas, by turning on the double trace interaction at a certain time (concretely speaking, at $\tau_{i}=0$ ) and by adjusting its strength $h$ appropriately as

$$
h=\bar{\phi} \frac{4 \pi}{\Delta B(\Delta, \Delta)}\left(\frac{L}{\ell^{2}}\right)^{2(1-\Delta)},
$$

the ETW configuration can be joined with the black hole configuration. Explicitly, the dilaton field can be obtained as

$$
\phi=\bar{\phi} L \frac{\cos \tau}{\cos \mu}+\varphi_{\mathrm{TW}}\left(u, v ; q_{i}=\pi / 4 \mid L\right),
$$

where we have taken $\phi_{\mathrm{hom}}=\phi_{\mathrm{BH}}$ and $\varphi_{\mathrm{TW}}$ denotes a specific combination of $\varphi_{\mathbf{I}}\left(u, v ; q_{i}\right)$ according to the value of $q_{i}$ (see eq. (4.13) in [20]). Here, $\varphi_{\mathbf{I}}\left(u, v ; q_{i}\right)$ is given by

$$
\varphi_{\mathbf{I}}\left(u, v ; q_{i}\right)=\ell^{2} \frac{\bar{h} \Delta N_{\Delta}}{2^{2 \Delta-2}} \int_{q_{i}}^{u} d q\left[w^{1-\Delta}(1-w)^{2 \Delta-1}+\Delta \frac{1+w}{1-w} B_{w}(1-\Delta, 2 \Delta)\right] .
$$


Under our gauge choice, through the asymptotic expansion of the dilaton field $\phi$, it turns out that the boundary proper time coordinates $\tilde{u}$ is related to the bulk global time $\tau$ as

$$
\tilde{u}=\theta(-\tau) \frac{\ell^{2}}{L} \operatorname{arctanh} \sin \tau+\theta(\tau) \frac{\ell^{2}}{L} \tau \quad(\tau>-\pi / 2),
$$

which shows a transition from black holes to ETWs around the point $\tau=0$. In the following, we consider sending signals on this black hole/ETW transition configuration. To see the bulk propagation of signal, we perturb the right system by turning on a source term to the total action by

$$
L=L_{\mathrm{TW}}+\gamma s(\tilde{u}) O_{\tilde{\Delta}}^{R}(\tilde{u}),
$$

where $L_{\mathrm{TW}}$ denotes the boundary action for two nearly $\mathrm{CFT}_{1}$ 's dual to the bulk gravity theory with the double trace deformation as

$$
L_{\mathrm{TW}}=L_{L}+L_{R}+h O_{\Delta}^{L}(\tilde{u}) O_{\Delta}^{R}(\tilde{u}) .
$$

One may recall that $L_{\mathrm{TW}}$ could be thought as the appropriate low energy (nearly $\mathrm{CFT}_{1}$ ) limit of two coupled SYK models ${ }^{1}$.

If the bulk starts with the two-sided black hole spacetime, the corresponding boundary system should be prepared at some initial time $\tilde{u}_{I}(<0)$ in a particularly entangled state, more specifically, a so-called thermofield double state. Basically here we are following the general correspondence between the two-sided black hole spacetime and the thermofield double description of the L-R boundary system [19, 26]. In this correspondence, the initial state is given by

$$
\left|\Psi\left(\tilde{u}=\tilde{u}_{I}\right)\right\rangle=\frac{1}{\sqrt{Z}} \sum_{n} e^{-\left(\frac{\beta}{2}+2 i \tilde{u}_{I}\right) E_{n}}|n\rangle \otimes|n\rangle
$$

where $\sqrt{Z}$ is the normalization factor and the inverse temperature $\beta$ is an inverse of the Gibbons-Hawking temperature

$$
\beta=\frac{1}{T}=\frac{2 \pi \ell^{2}}{L} .
$$

Note that the $\mathrm{L}$ and $\mathrm{R}$ systems (without L-R interactions) possess an identical (nearly) $\mathrm{CFT}_{1}$ Hamiltonian $H$ leading to identification $H_{L} / H_{R}=H \otimes 1 / 1 \otimes H$, respectively. $E_{n}$ and $|n\rangle$ are denoting eigenvalues and eigenstates of the Hamiltonian $H$. Thus initially the left and right boundary systems are maximally entangled for a given temperature $T$ and further the phase factor of each $|n\rangle \otimes|n\rangle$ should be arranged initially as given in the above. The subsequent time evolution for the two-sided black hole system is given in terms of total Hamiltonian $H_{\text {total }}=H_{L}+H_{R}$. When an additional interaction is turned on, of course, the total Hamiltonian of the system becomes $H_{\text {total }}=H_{L}+H_{R}+H_{\text {int }}$ with $H_{\text {int }}=\delta H$ in (2.11) e.g. for the black hole/ETW transitional configuration. When the transition occurs at $\tilde{u}_{i}=0$ as above, we note that the thermofield state at $\tilde{u}=0$ can be approximated to the ground state of the ETW system [25] and the subsequent evolution after the transition is almost stationary.

\footnotetext{
${ }^{1}$ The parameter $g$ in the Schwarzian theory could be written in terms of the SYK model variables as $\bar{\phi}=\frac{\mu \alpha_{S}}{\mathcal{J}}$ and $\frac{g}{2^{2 \Delta}}\left(\frac{N}{\phi_{R}}\right)^{2 \Delta-1}=\frac{\mu \alpha_{S}}{\mathcal{J}} \frac{c_{\Delta}}{\left(2 \alpha_{S}\right)^{2 \Delta}}$ (see [25] for this identification).,Maldacena:2017axo,Maldacena:2018lmt
} 


\section{Signaling in traversable wormhole}

In this section, we shall clarify signal propagations in our traversable wormhole systems. See $[27,28]$ for some related aspects. Of course, without the double trace deformation, the L-R systems are completely disconnected from each other and there is no way to send any signal from one side to the other. In the bulk of the black hole spacetime, a signal from one boundary enters the horizon and hits the singularity before meeting the other side. Namely the wormhole is not traversable in this case.

Once the L-R interaction is turned on, the wormhole becomes traversable. In this traversable wormhole system, there are generically two channels of the information propagation. ${ }^{2}$ One is the channel through the bulk. This respects the bulk causality and takes a certain amount of time for a signal transfer. This can be used for the quantum teleportation sending quantum states through the bulk. The other channel is through the direct interaction which is instantaneous as the left and the right boundary times are identified and consequently interaction requires no time delay. Let us clarify these two channels respectively beginning with the bulk signaling.

To this end, we turn on the source term on the right side (or the left side if one wishes) for an operator of dimension $\tilde{\Delta}$ as given in (2.23). We shall switch on the source term only for $\tilde{u} \in\left[\tilde{u}_{i}, \tilde{u}_{f}\right]$, so $s(\tilde{u})$ vanishes when $\tilde{u}<\tilde{u}_{i}$ or $\tilde{u}>\tilde{u}_{f}$. First, let us recall that the bulk to boundary two point functions are given by

$$
\begin{aligned}
& K_{L}\left(\tau-\tau_{s}, \mu ; \Delta\right)=\mathcal{N}_{\Delta}\left[\frac{\cos \mu}{\cos \left(\tau-\tau_{s}\right)+\sin \mu}\right]^{\Delta}, \\
& K_{R}\left(\tau-\tau_{s}, \mu, \Delta\right)=\mathcal{N}_{\Delta}\left[\frac{\cos \mu}{\cos \left(\tau-\tau_{s}\right)-\sin \mu}\right]^{\Delta},
\end{aligned}
$$

where

$$
\mathcal{N}_{\Delta}=\frac{2^{\Delta-2} \Gamma^{2}(\Delta)}{\pi \Gamma(2 \Delta)}
$$

Here, $L / R$ represent that the locations of the relevant boundary sources, which are taken, in global $(\tau, \mu)$ coordinates, as $\left(\tau_{s},-\frac{\pi}{2}\right)$ for $K_{L}$ and as $\left(\tau_{s}, \frac{\pi}{2}\right)$ for $K_{R}$, respectively. Since the exponent $\Delta$ is not an integer in our case, the phase of $K_{L / R}$ functions should be chosen appropriately, whenever the values inside brackets are negative.

Since we turn on the right side source, we use $K_{R}$ to construct the corresponding bulk solution. The retarded condition requires $\tau-\tau_{s}>-\mu+\frac{\pi}{2}$. The corresponding scalar field is solved by

$$
\chi=\gamma \int_{\tau_{i}}^{\tau_{f}} d \tau_{s} \tilde{s}\left(\tau_{s}\right) K\left(\tau-\tau_{s}, \mu ; \tilde{\Delta}\right)
$$

where

$$
\tilde{s}\left(\tau_{s}\right)=s\left(\tilde{u}\left(\tau_{s}\right)\right)\left(\tau_{s}^{\prime}\right)^{\tilde{\Delta}-1} .
$$

\footnotetext{
${ }^{2}$ We thank Juan Maldacena for the discussions and clarification on this point.
} 
The retarded Green function $K$ is given $b^{3}$

$$
\begin{aligned}
K(\tau, \mu ; \Delta) & =i\left(K_{R}(\tau-i \epsilon, \mu ; \Delta)-K_{R}^{*}(\tau+i \epsilon, \mu ; \Delta)\right) \theta\left(\tau-\tau_{s}\right) \\
& =2 \sin \nu_{R}\left|K_{R}(\tau, \mu ; \Delta)\right| \theta\left(\tau-\tau_{s}+\mu-\frac{\pi}{2}\right)
\end{aligned}
$$

where $\nu_{R}$ refers to the phase as in $K_{R}^{*}(\tau+i \epsilon)=e^{i \nu_{R}}\left|K_{R}^{*}(\tau+i \epsilon)\right|$.

At this stage, it would be useful to provide some details for the second equality in (3.5). This is related to the phase choice or the choice of branch cut and appropriate sheet in the multi-valued function $f(x)=x^{\Delta}$ in the bulk to boundary function $K_{R}$.

If we ignore the multi-valuedness of the bulk to boundary function $K_{R}$ in (3.1), it is $2 \pi$ periodic with respect to $\tau-\tau_{s}$, and then the evaluation point $(u, v)$ would be space-like separated from the source point $\left(\tau_{s}, \frac{\pi}{2}\right)$, as can be seen from figure 1 . This naive periodicity give us the incorrect physical picture, since the evaluation point $(u, v)$ should be time-like separated from the source point $\left(\tau_{s}, \frac{\pi}{2}\right)$. In order to rectify this unwilling aspect, we need to introduce appropriate phase factors in $K_{R}$ (or $K_{R}^{*}$ ) in such a way that the evaluation point $(u, v)$ is time-like separated from the source point. In other words, we need to choose appropriate sheet for the correct value of the multi-valued function $f(x)=x^{\Delta}$. From the causality consideration, the appropriate phase choice in $K_{R}^{*}$ would be

$$
\left[\frac{\cos \mu}{\cos \left(\tau-\tau_{s}\right)-\sin \mu}\right]^{\Delta} \rightarrow\left[-\frac{\cos \mu}{\cos \left(\tau-\tau_{s}\right)-\sin \mu}\right]^{\Delta}=e^{i \pi \Delta}\left[\frac{\cos \mu}{\cos \left(\tau-\tau_{s}\right)-\sin \mu}\right]^{\Delta} .
$$

The additional phase $e^{i \pi \Delta}$ should be taken into consideration whenever we go across $+45^{\circ}$ red lines from yellow to green triangle regions in an upward direction and vice versa. Equipped with these additional phases one can obtain the final expression in (3.5).

After these considerations, we obtain the phase assignments of $\nu_{R}$ in figure 1 as follows: ${ }^{4}$

$$
\nu_{R}\left(\tau-\tau_{s}, \mu ; \Delta\right)=\left\{\begin{array}{ll}
(2 n+1) \pi \Delta & \text { if } \quad \mu+\left(2 n+\frac{1}{2}\right) \pi<\tau-\tau_{s}<\mu+\left(2 n+\frac{3}{2}\right) \pi \\
2 n \pi \Delta & \text { if } \quad \mu+\left(2 n-\frac{1}{2}\right) \pi<\tau-\tau_{s}<-\mu+\left(2 n+\frac{1}{2}\right) \pi
\end{array} .\right.
$$

We illustrate this phase assignment on the right of figure 1.

In the asymptotic region of the left boundary $\mu \sim-\pi / 2$, the leading order of the scalar field behaves as

$$
\chi=O\left(\cos ^{\tilde{\Delta}} \mu\right) .
$$

Hence, no source term is present in the left side while the expectation value of operator $O_{L}^{\tilde{\Delta}}$ is induced by the bulk transmission. The induced expectation value is given by

$$
\left\langle O_{L}(\tau)\right\rangle=2 \mathcal{N}_{\tilde{\Delta}} \gamma \int_{\tau_{i}}^{\tau_{f}} d \tau_{s} \tilde{s}\left(\tau_{s}\right)\left[\frac{1}{1+\cos \left(\tau-\tau_{s}\right)}\right]^{\tilde{\Delta}} \theta\left(\tau-\tau_{s}-\pi\right) \sin \nu_{L R}
$$

with $\nu_{L R}$ denoting $\nu_{R}\left(\tau-\tau_{s} ;-\pi / 2 ; \tilde{\Delta}\right)$. The factor in the square bracket becomes infinity when $\tau-\tau_{s}=(2 n+1) \pi$ with integer $n$. Therefore, the information on the left side appears

\footnotetext{
${ }^{3}$ We have taken the conventional retarded Green function as $G_{\text {ret }}\left(x, x^{\prime}\right) \equiv i\left\langle\left[\chi(x), \chi\left(x^{\prime}\right)\right]\right\rangle \theta\left(\tau-\tau^{\prime}\right)$, which satisfies $\left(-\square+m^{2}\right) G_{\text {ret }}(x)=\delta(x)$.

${ }^{4}$ This phase assignment is already made in our previous work [20].
} 

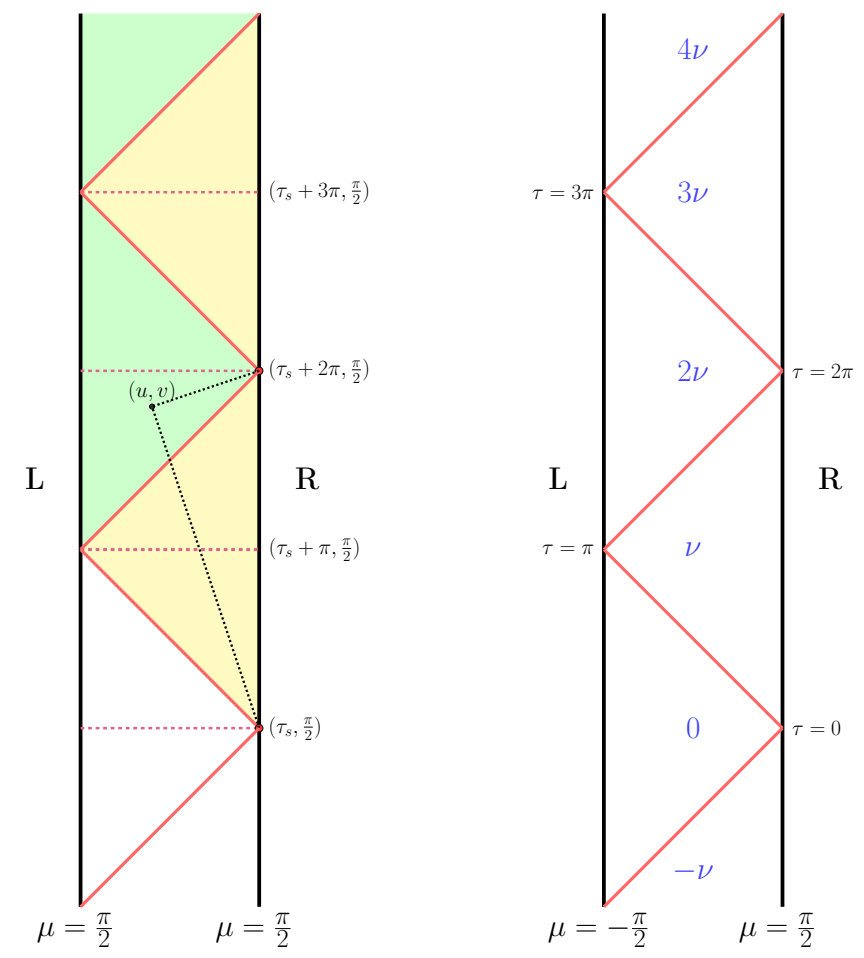

Figure 1. On the left, the evaluation point $(u, v)$ should be timelike separated from the source point $\left(\tau_{s}, \frac{\pi}{2}\right)$, while the naive $2 \pi$ periodicity implies that it is spacelike separated from the source. On the right, the phase assignment for $K_{R}^{*}$ is given where $\nu=\pi \Delta$ with $\tau_{s}=0$.

only when $\tau>\tau_{s}+\pi$ and the corresponding propagation respects bulk causality. Here, we also record the expectation value induced on the right side given by

$$
\left\langle O_{R}(\tau)\right\rangle=2 \mathcal{N}_{\tilde{\Delta}} \gamma \int_{\tau_{i}}^{\tau_{f}} d \tau_{s} \tilde{s}\left(\tau_{s}\right)\left[\frac{1}{1-\cos \left(\tau-\tau_{s}\right)}\right]^{\tilde{\Delta}} \theta\left(\tau-\tau_{s}\right) \sin \nu_{R R}
$$

with $\nu_{R R}$ denoting $\nu_{R}\left(\tau-\tau_{s} ; \pi / 2 ; \tilde{\Delta}\right)$. Note that the factor in the square bracket in this RR case becomes infinity when $\tau-\tau_{s}=2 n \pi$ with integer $n$.

One may perform the corresponding CFT analysis. There the propagator from the right source to the expectation value on the left is simply given by

$$
k_{L R}\left(\tau-\tau_{s} ; \tilde{\Delta}\right)=2 \mathcal{N}_{\tilde{\Delta}}\left[\frac{1}{1+\cos \left(\tau-\tau_{s}\right)}\right]^{\tilde{\Delta}} \theta\left(\tau-\tau_{s}-\pi\right) \sin \nu_{L R},
$$

which is following from the nearly conformal symmetry of the traversable wormhole system. Then from this the above result can be regained straightforwardly.

We now turn to the discussion of the other channel, which may be more clearly seen in the Schwarzian description in (2.12). In this system, we consider a perturbation on the right side as $\delta \tau_{R} \cdot{ }^{5}$ The left side $\tau_{L}$ will then be instantaneously affected by the perturbation

\footnotetext{
${ }^{5}$ In the SYK model, $\tau_{R}$ and $\tau_{L}$ corresponds to the reparametrization modes of $\mathrm{L} / \mathrm{R}$ system which are the soft modes of the nearly CFT systems.
} 


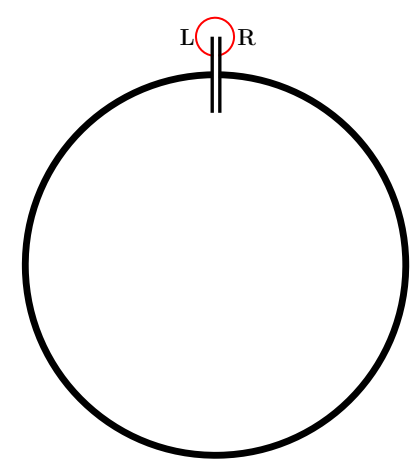

Figure 2. The spatial part of the Lorentzian traversable wormhole system is depicted schematically. One channel of signal propagation is through the bulk, which takes a certain amount of time due to the bulk causality. The other is the direct propagation through the interaction between the L-R systems, which is instantaneous.

of $\tau_{R}$, which is basically due to the L-R interaction term, as may be seen as follows. By taking into account the linear perturbation of the Schwarzian action in eq. (2.12), one can obtain

$$
\frac{2}{\tau_{g}^{\prime 4}} \delta \tau_{L}^{\prime \prime \prime \prime}+\frac{2(2-\Delta)}{\tau_{g}^{\prime 2}} \delta \tau_{L}^{\prime \prime}+\delta \tau_{L}=\frac{2 \Delta}{\tau_{g}^{\prime 2}} \delta \tau_{R}^{\prime \prime}+\delta \tau_{R}, \quad ' \equiv \frac{d}{d \tilde{u}},
$$

where $\tau_{g}^{\prime}$ denotes the constant corresponding to the eternal traversable wormhole solution chosen $^{6}$ as $\tau_{L}^{\prime}=\tau_{R}^{\prime}=\tau_{g}^{\prime}$. This shows us the instantaneous reaction of $\tau_{L}$ by the change of $\tau_{R}$ in the amount of $\delta \tau_{R}$.

These two channels are schematically depicted in figure 2. The big circle represents the bulk traversable wormhole and small red circle represents the instantaneous boundary channel directly through the interactions. Our wormhole system can be embedded into higher dimensions [15] (see also [29]). In this case, the L and $\mathrm{R}$ systems can be spatially separated in the boundary spacetime. Of course, the boundary signal propagation through the boundary interactions takes some amount of time depending on the separation. Then the overall causality requires that the bulk propagation through the wormhole should not be faster than the boundary propagation, as dictated by the causality of the boundary theory. Interestingly, this is consistent with the Gao-Wald theorem [30, 31], even though some conditions for the theorem are violated in our setup.

In the next section, we shall discuss a possible experimental probe of the bulk channel purely in terms of boundary operations. This discussion is focused on the characteristics of the bulk channel that can also be used for the quantum teleportation.

\section{Experimental probes of wormholes}

In the L-R boundary systems, suppose that signals are sent from the $\mathrm{R}$ system and the observer in the L system detects them. The observer may wonder from which channel they

\footnotetext{
${ }^{6}$ The constant $\tau_{g}^{\prime}$ value could be related to $\beta_{g}$, which would be introduced later in eq. (4.5), as $\tau_{g}^{\prime}=\frac{2 \pi}{\beta_{g}}$. Note also that there is an additional equation, in which $\tau_{L}$ and $\tau_{R}$ are interchanged on the linear perturbation of the Schwarzian action.
} 
emerged. The boundary channel would always be an obvious candidate since, without any boundary interaction, the two systems are completely disconnected. Then it would be more interesting to ask whether there is any situation that the L observer would naturally conclude that the detected signal must have passed not through the usual boundary channel but through other channel, i.e., the bulk channel. Note that from the pure boundary viewpoint, the existence of the bulk itself is nontrivial and also a boundary interaction does not always guarantee traversability of the bulk channel.

To answer this question, let us consider the configuration (2.20), which describes the black hole/ETW transition as depicted on the left panel of figure 3, dubbed as transparentization of a black hole to an ETW [20]. The boundary time $\tilde{u}$ is then related to the bulk global time as (2.22). In this configuration, one may send a signal through the bulk from one to the other side with perturbation (2.23). The boundary time $\tilde{u}_{o}$ that the signal sent at $\tilde{u}_{s}$ is observed at the other side is given by

$$
\tilde{u}_{o}=\frac{\beta}{2}+\theta\left(\tilde{u}_{s}\right) \tilde{u}_{s}+\frac{\beta}{2 \pi} \theta\left(-\tilde{u}_{s}\right) \arcsin \tanh \frac{2 \pi \tilde{u}_{s}}{\beta} .
$$

As discussed in [20], the signals sent during $-\infty<\tilde{u}_{s}<0$, which is before turning on the boundary interaction, would come out at the other side during the time $\frac{\beta}{4}<\tilde{u}_{o}<\frac{\beta}{2}$, obeying the bulk causality. Moreover, they are blue-shifted. If the signals are sent with frequency $\omega_{s}$ which is controlled by the source term $s(\tilde{u})$ in $(2.23)$, the frequency $\omega_{o}$ observed at the other side is given by

$$
\omega_{o}=\omega_{s} \cosh \frac{2 \pi \tilde{u}_{s}}{\beta} .
$$

Note that the signal sent earlier would be blue-shifted more strongly. For example, the blue-shift factor is 268 for $\tilde{u}_{s}=-\beta$, while it is as high as 143376 for $\tilde{u}_{s}=-2 \beta$.

On the other hand, it is obvious that signals from the boundary channel would emerge to the other side instantaneously without any blue-shift factor. Therefore, even though there are two channels of signal propagation, they could be clearly distinguished in the black hole/ETW transitional configuration.

It might be the case that experimentally realizing the particular set of phases appearing in the thermofield initial state in (2.25) is not that straightforward. To avoid such complications, one may turn on the L-R interaction at $\tilde{u}=\tilde{u}_{i}>0$ and consider the system from $\tilde{u}_{I}=0$ in which the initial state does not involve any such phases. The corresponding Schwarzian dynamics is described by

$$
L=\bar{\phi}\left(\frac{\tau^{\prime \prime}}{\tau^{\prime}}\right)^{2}-\bar{\phi}\left(\tau^{\prime}\right)^{2}+\frac{g}{2^{2 \Delta}}\left(\tau^{\prime}\right)^{2 \Delta} \theta\left(\tilde{u}-\tilde{u}_{i}\right)
$$

where we set $\tau_{L}=\tau_{R}=\tau$ as before. ${ }^{7}$ We begin with a black hole with an inverse temperature $\beta$ described by a solution $\sin \tau=\tanh \frac{2 \pi \tilde{u}}{\beta}$ for $\tilde{u}<\tilde{u}_{i}$. At $\tilde{u}=\tilde{u}_{i}$ where the interaction is turned on, $\tau^{\prime}$ and $\tau^{\prime \prime}$ should be continuous. We require $E_{i}<0$ right after

\footnotetext{
${ }^{7}$ Normally we take $\mathcal{Y}=\log \tau^{\prime}$ as our dynamical variable and then the kinetic term is given by $K=\bar{\phi}\left(\mathcal{Y}^{\prime}\right)^{2}$ together with the potential $V=\bar{\phi} e^{2 \mathcal{Y}}-\frac{g}{2^{2 \Delta}} e^{2 \Delta \mathcal{Y}} \theta\left(\tilde{u}-\tilde{u}_{i}\right)$.
} 

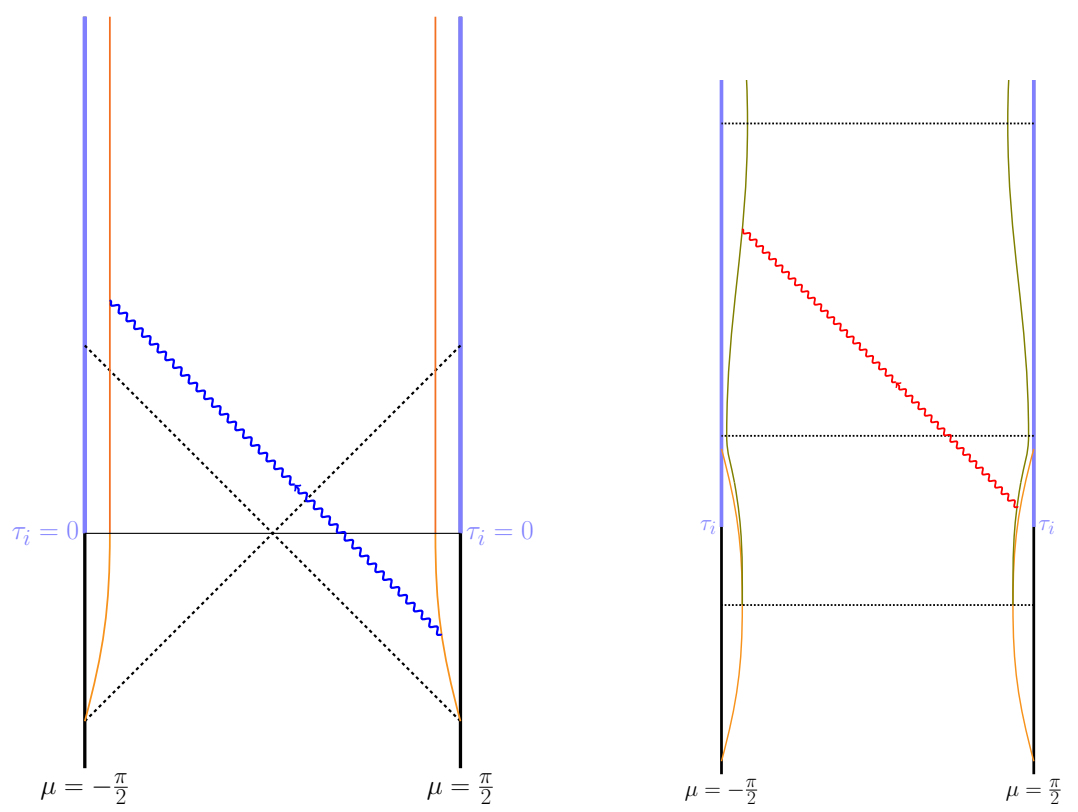

Figure 3. On the left we depict a transitional configuration of the black hole to ETW where the L$\mathrm{R}$ boundary interaction is turned on at $\tilde{u}_{i}=0$. On the right we depict a transitional configuration of the black hole to an excited (oscillatory) ETW state where the L-R boundary interaction is turned on at $\tilde{u}_{i}>0$. Signals sent before turning on the L-R interaction through the bulk channel emerges to the other side after some amount of time with modulated frequencies, which is a clear evidence of the existence of the bulk channel and traversability.

the interaction is turned on in order to have a bound state motion $\left(\tau^{\prime}>0\right)$ leading to a traversable wormhole state. This then gives a condition

$$
\Delta \cosh ^{2 \Delta} \frac{2 \pi \tilde{u}_{i}}{\beta}<\left(\frac{\beta}{\beta_{g}}\right)^{2(1-\Delta)},
$$

where we introduce a length scale $\beta_{g}$ by

$$
\beta_{g}=2 \pi\left(\frac{g \Delta}{2^{2 \Delta} \bar{\phi}}\right)^{-\frac{1}{2(1-\Delta)}}
$$

The subsequent time evolution with variable $\tau^{\prime}$ satisfies the equation of motion

$$
E_{i}=\bar{\phi}\left[\left(\frac{\tau^{\prime \prime}}{\tau^{\prime}}\right)^{2}+\bar{\phi}\left(\tau^{\prime}\right)^{2}\right]-\frac{g}{2^{2 \Delta}}\left(\tau^{\prime}\right)^{2 \Delta}
$$

as depicted in figure 4. This wormhole configuration is in an excited state of the ETW system and becomes oscillatory as depicted on the right side panel of figure 3. Note that the L/R boundary trajectories (green curves in the right panel in figure 3 ) are determined by the equations

$$
\mu(\tilde{u})=\mp\left[\frac{\pi}{2}-\epsilon \tau^{\prime}(\tilde{u})+\mathcal{O}\left(\epsilon^{2}\right)\right]
$$




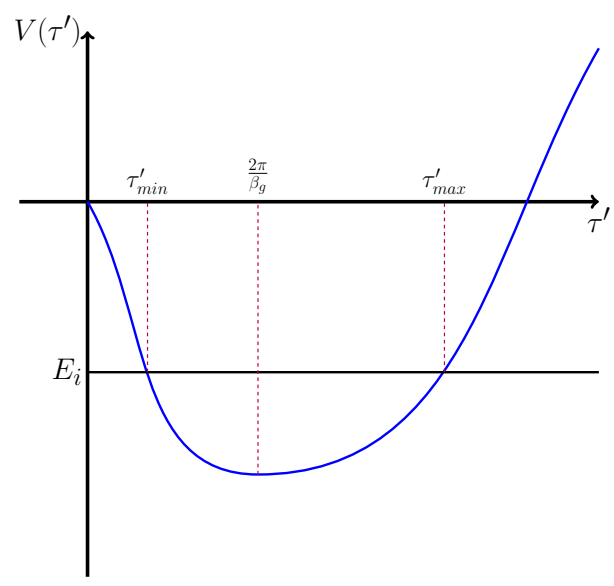

Figure 4. The potential as a function of $\tau^{\prime}$ is depicted in this figure. We also indicate the bounded motion and its energy which is negative.

We now send a signal from the right at $\tilde{u}=\tilde{u}_{s}$ with $0<\tilde{u}_{s}$. One finds the signal through the wormhole appears on the left side when $\tilde{u}_{o}$ determined by the condition $\tau\left(\tilde{u}_{o}\right)=$ $\tau\left(\tilde{u}_{s}\right)+\pi$. Thus again there is the corresponding delay and the frequency modulation of signal is given by

$$
\omega_{0}=\omega_{s} \frac{\tau^{\prime}\left(\tilde{u}_{o}\right)}{\tau^{\prime}\left(\tilde{u}_{s}\right)}
$$

which may be either red-shifted or blue-shifted depending on $\tilde{u}_{s}$ and the parameter $\beta_{g}$. In appendices A and B, we present some detailed analysis of this bulk signaling problem for two specific cases where the L-R interaction is turned on at $\tilde{u}_{i}>0$.

Finally, one may also consider the ETW/black hole transitional configuration given in [20] where its transition is now induced by turning off the L-R interaction at $\tilde{u}_{i}=0$. In this case, one finds again a delay together with a modulation of the signal frequency. Its experimental realization is expected to be relatively simple since our system in this case starts in the ground state of the ETW system which is stationary until the transition.

In condensed matter physics, there are several attempts to engineer the SYK model with ultracold gases, graphene flakes, quantum wires or 3D topological insulators (see e.g. [32] and references therein). Then it might be possible to experimentally realize the holographic dual of the traversable wormhole system by entangling two SYK model systems. By turning on or off different type of interactions between them, one may send signals from one side to the other. If frequency-modulated signals are detected after some amount of time delay for a double trace interaction while no such signals are observed for other cases, it could be considered as a strong evidence that the bulk is real and a traversable wormhole is formed between the two systems.

\section{Conclusions}

In this note, we clarify the nature of signal propagation in traversable wormhole system. Basically we show that there are two independent channels. One is through the bulk worm- 
hole and the other is the direct boundary channel which is via the boundary interaction. We also describe in detail how the information can be transferred from one side to the other through the bulk wormhole using a bulk scalar field dual to a boundary scalar operator.

We then take the examples of the bulk representing the transition from the black hole from/to the ETW geometry where the transition is induced by turning off/on the double trace deformation. The signal from one side emerges to the other side after certain amount of time perfectly respecting the bulk causality. The signal frequency detected on the other side will be in general highly modulated while passing through the bulk of traversable wormhole. These two facts may be used to show the existence of the bulk wormhole since the frequency-modulation patterns as well as the time delay imprint clear characteristics of the bulk geometry. Also note that, in this measurement, one needs to perform a simple set of purely boundary operations only.

The experimental realization of our setup in the boundary system seems feasible without any fundamental difficulties such as probing the behind horizon degrees in ordinary two-sided black hole systems.

\section{Acknowledgments}

We thank Juan Maldacena for enlightening discussions for clarifying the nature of interactions in traversable wormhole systems. DB was supported in part by NRF Grant 2017R1A2B4003095 and by Basic Science Research Program through National Research Foundation funded by the Ministry of Education (2018R1A6A1A06024977). C.K. was supported by NRF Grant 2019R1F1A1059220. S.-H.Y. was supported by NRF Grant 2018R1D1A1A09082212.

\section{A Perturbative solution to ETW}

In this appendix we provide some details on the signal sending on the configuration in the right panel of figure 3. This configuration could be described by adjoining the black hole solution for $\tilde{u} \leq \tilde{u}_{i}$ to a traversable wormhole solution for $\tilde{u}>\tilde{u}_{i}$. In the context of the Schwarzian dynamics in (4.3), we consider a perturbative solution around the ETW solution $\tau^{\prime}=\frac{2 \pi}{\beta_{g}}$ (see figure 4 ) for $\tilde{u}>\tilde{u}_{i}$, by taking

$$
\tau^{\prime}(\tilde{u})=\frac{2 \pi}{\beta_{g}}+\delta \tau^{\prime}(\tilde{u})
$$

with the Lagrangian in (4.3) as

$$
L=\bar{\phi}\left(\frac{2 \pi}{\beta_{g}}\right)^{2} \frac{1-\Delta}{\Delta}+\bar{\phi}\left(\frac{\beta_{g}}{2 \pi}\right)^{2}\left[\left(\delta \tau^{\prime \prime}\right)^{2}-\omega_{g}^{2}\left(\delta \tau^{\prime}\right)^{2}+\cdots\right] \quad \text { for } \quad \tilde{u}>\tilde{u}_{i} .
$$

Here, $\omega_{g}$ is defined by

$$
\omega_{g} \equiv \frac{2 \pi}{\beta_{g}} \sqrt{2(1-\Delta)}
$$


To match the perturbative solution for $\tilde{u}>\tilde{u}_{i}$ with the black hole solution for $\tilde{u} \leq \tilde{u}_{i}$ at $\tilde{u}=\tilde{u}_{i}$, we take the parameters in such a way that $\frac{2 \pi}{\beta}\left|\tilde{u}_{i}\right| \ll 1$ and $\left|\frac{\beta}{\beta}{ }_{g}-1\right| \ll 1$. Then, the matched solution is given by

$$
\tau^{\prime}(\tilde{u})=\left\{\begin{array}{ll}
\frac{2 \pi}{\beta} \frac{1}{\cosh \frac{2 \pi}{\beta} \tilde{u}} & \text { for } \tilde{u} \leq \tilde{u}_{i} \\
\frac{2 \pi}{\beta_{g}}-\frac{2 \pi}{\beta_{g}}\left[a \cos \omega_{g} \tilde{u}+\left(\frac{2 \pi}{\beta_{g}}\right)^{2} \frac{\tilde{u}_{i}}{\omega_{g}} \sin \omega_{g} \tilde{u}\right]+\cdots & \text { for } \tilde{u}>\tilde{u}_{i}
\end{array},\right.
$$

where $a$ is defined by $a \equiv \frac{\beta}{\beta_{g}}-1$ and $\cdots$ denotes higher order terms in $a$ and/or $\frac{2 \pi}{\beta} \tilde{u}_{i}$.

Now, let us consider a signal which is sent at $\tilde{u}=\tilde{u}_{s}$ in such a way that $\left|\tilde{u}_{s}\right|<\left|\tilde{u}_{i}\right|$. Since $\frac{2 \pi}{\beta}\left|\tilde{u}_{s}\right|<\frac{2 \pi}{\beta}\left|\tilde{u}_{i}\right| \ll 1$, one can see that

$$
\tau\left(\tilde{u}_{s}\right)=\frac{2 \pi}{\beta} \tilde{u}_{s} .
$$

Let this signal be observed at $\tilde{u}_{o}$, which is realized in the bulk global time as $\tau\left(\tilde{u}_{o}\right)=$ $\pi+\tau\left(\tilde{u}_{s}\right)$. By using the perturbative solution for $\tilde{u}>\tilde{u}_{i}$, one can also see that

$$
\tau\left(\tilde{u}_{o}\right)=\frac{2 \pi}{\beta_{g}} \tilde{u}_{o}-\frac{2 \pi}{\beta_{g} \omega_{g}}\left[a \sin \omega_{g} \tilde{u}_{o}-\left(\frac{2 \pi}{\beta_{g}}\right)^{2} \frac{\tilde{u}_{i}}{\omega_{g}}\left(\cos \omega_{g} \tilde{u}_{o}-1\right)\right]+\cdots,
$$

where we have chosen the initial condition as $\tau(\tilde{u}=0)=0$. As a result, one obtains

$$
\begin{aligned}
\tilde{u}_{o}-\tilde{u}_{s} & =\frac{\beta_{g}}{2}+\frac{a}{\omega_{g}} \sin \frac{\beta_{g} \omega_{g}}{2}+2\left(\frac{2 \pi}{\beta_{g}}\right)^{2} \frac{\tilde{u}_{i}}{\omega_{g}^{2}} \sin ^{2} \frac{\beta_{g} \omega_{g}}{4}+\cdots, \\
\omega_{o} & =\omega_{s}\left[1+2 a \sin ^{2} \frac{\beta_{g} \omega_{g}}{4}-\left(\frac{2 \pi}{\beta_{g}}\right)^{2} \frac{\tilde{u}_{i}}{\omega_{g}} \sin \frac{\beta_{g} \omega_{g}}{2}+\cdots\right],
\end{aligned}
$$

where $\omega_{s}$ and $\omega_{o}$ are the frequencies at $\tilde{u}_{s}$ and $\tilde{u}_{o}$, respectively. For purposes of comparison, one may notice that the result in (4.1) becomes, under the present assumption that $\frac{2 \pi}{\beta} \tilde{u}_{s} \ll 1$,

$$
\tilde{u}_{o}=\frac{\beta}{2}+\tilde{u}_{s},
$$

which is consistent with the above result up to the relevant order. This result shows us clearly that the frequency modulation of the signal could be either blue-shifted or redshifted according to the parameter choice.

\section{B $\Delta=\frac{1}{2}$ case}

In this appendix we consider a special case of $\Delta=\frac{1}{2}$ in (4.3). In this case the bounded motion for $\tilde{u}>\tilde{u}_{i}$ would have a conserved energy as

$$
H=\bar{\phi}\left[\left(\frac{\tau^{\prime \prime}}{\tau^{\prime}}\right)^{2}+\tau^{\prime 2}-\frac{g}{2 \bar{\phi}} \tau^{\prime}\right]=E_{i},
$$

which gives us

$$
\frac{\tau^{\prime \prime}}{\tau^{\prime}}= \pm \sqrt{\left(\tau^{\prime}-\tau_{\min }^{\prime}\right)\left(\tau_{\max }^{\prime}-\tau^{\prime}\right)}
$$


Here, $\tau_{\min / \max }^{\prime}$ denotes the turning points of the bounded motion (see figure 4 ) and are related to the parameters as

$$
\frac{1}{2}\left(\tau_{\min }^{\prime}+\tau_{\max }^{\prime}\right)=\frac{2 \pi}{\beta_{g}}=\frac{g}{4 \bar{\phi}}, \quad \bar{\phi} \tau_{\min }^{\prime} \tau_{\max }^{\prime}=-E_{i} .
$$

Integrating the above differential equation, one can see that the solution for the bounded motion is given by

$$
\tau^{\prime}(\tilde{u})=\frac{2 \tau_{\max }^{\prime} \tau_{\min }^{\prime}}{\left[\tau_{\max }^{\prime}+\tau_{\min }^{\prime}-\left(\tau_{\max }^{\prime}-\tau_{\min }^{\prime}\right) \sin \sqrt{\tau_{\max }^{\prime} \tau_{\min }^{\prime}}\left(\tilde{u}-\tilde{u}_{c}\right)\right]},
$$

where $\tilde{u}_{c}$ is an integration constant. By integrating this expression, one obtains finally

$$
\tan \left[\frac{\tau-\tau_{c}}{2}\right]=\frac{\tau_{\max }^{\prime}+\tau_{\min }^{\prime}}{2 \sqrt{\tau_{\max }^{\prime} \tau_{\min }^{\prime}}} \tan \left[\frac{\sqrt{\tau_{\max }^{\prime} \tau_{\min }^{\prime}}}{2}\left(\tilde{u}-\tilde{u}_{c}\right)\right]-\frac{\tau_{\max }^{\prime}-\tau_{\min }^{\prime}}{2 \sqrt{\tau_{\max }^{\prime} \tau_{\min }^{\prime}}},
$$

where $\tau_{c}$ is another integration constant.

As before, one can fix the constants by matching the solution to the black hole solution at $\tilde{u}=\tilde{u}_{i}$ by using the continuity of $\tau^{\prime}$ and $\tau^{\prime \prime}$. And then, it is straightforward to consider the time delay and frequency modulation for the signal sent at $\tilde{u}=\tilde{u}_{s}$, at least numerically.

Open Access. This article is distributed under the terms of the Creative Commons Attribution License (CC-BY 4.0), which permits any use, distribution and reproduction in any medium, provided the original author(s) and source are credited.

\section{References}

[1] R. Jackiw, Lower dimensional gravity, Nucl. Phys. B 252 (1985) 343 [InSPIRE].

[2] C. Teitelboim, Gravitation and Hamiltonian structure in two space-time dimensions, Phys. Lett. B 126 (1983) 41.

[3] K. Jensen, Chaos in AdS $S_{2}$ holography, Phys. Rev. Lett. 117 (2016) 111601 [arXiv: 1605.06098] [INSPIRE].

[4] J. Engelsöy, T.G. Mertens and H. Verlinde, An investigation of $A d S_{2}$ backreaction and holography, JHEP 07 (2016) 139 [arXiv:1606.03438] [INSPIRE].

[5] J. Maldacena, D. Stanford and Z. Yang, Conformal symmetry and its breaking in two dimensional nearly Anti-de-Sitter space, PTEP 2016 (2016) 12C104 [arXiv:1606.01857] [INSPIRE].

[6] J. Maldacena, D. Stanford and Z. Yang, Diving into traversable wormholes, Fortsch. Phys. 65 (2017) 1700034 [arXiv: 1704.05333] [InSPIRE].

[7] D. Stanford and E. Witten, Fermionic localization of the Schwarzian theory, JHEP 10 (2017) 008 [arXiv: 1703.04612] [inSPIRE].

[8] P. Saad, S.H. Shenker and D. Stanford, JT gravity as a matrix integral, arXiv:1903.11115 [INSPIRE].

[9] D. Stanford and E. Witten, JT gravity and the ensembles of random matrix theory, arXiv: 1907.03363 [INSPIRE]. 
[10] T. Muta and S.D. Odintsov, Two-dimensional higher derivative quantum gravity with constant curvature constraint, Prog. Theor. Phys. 90 (1993) 247 [Phys. Atom. Nucl. 56 (1993) 1121] [Yad. Fiz. 56 (1993) 223] [INSPIRE].

[11] A. Kitaev, A simple model of quantum holography, talks given at KITP, April 7 and May 27 (2015).

[12] J. Maldacena, G.J. Turiaci and Z. Yang, Two dimensional nearly de Sitter gravity, arXiv:1904.01911 [INSPIRE].

[13] J. Cotler, K. Jensen and A. Maloney, Low-dimensional de Sitter quantum gravity, arXiv: 1905.03780 [INSPIRE].

[14] P. Gao, D.L. Jafferis and A.C. Wall, Traversable wormholes via a double trace deformation, JHEP 12 (2017) 151 [arXiv:1608.05687] [INSPIRE].

[15] J. Maldacena, A. Milekhin and F. Popov, Traversable wormholes in four dimensions, arXiv: 1807.04726 [INSPIRE].

[16] L. Susskind, Three lectures on complexity and black holes, arXiv:1810.11563 [INSPIRE].

[17] L. Susskind, Dear qubitzers, GR=QM, arXiv:1708.03040 [INSPIRE].

[18] J. Maldacena and L. Susskind, Cool horizons for entangled black holes, Fortsch. Phys. 61 (2013) 781 [arXiv:1306.0533] [INSPIRE].

[19] D. Bak, C. Kim and S.-H. Yi, Bulk view of teleportation and traversable wormholes, JHEP 08 (2018) 140 [arXiv: 1805.12349] [INSPIRE].

[20] D. Bak, C. Kim and S.-H. Yi, Transparentizing black holes to eternal traversable wormholes, JHEP 03 (2019) 155 [arXiv: 1901.07679] [INSPIRE].

[21] A. Almheiri and J. Polchinski, Models of AdS 2 backreaction and holography, JHEP 11 (2015) 014 [arXiv: 1402.6334] [INSPIRE].

[22] I.R. Klebanov and E. Witten, AdS/CFT correspondence and symmetry breaking, Nucl. Phys. B 556 (1999) 89 [hep-th/9905104] [INSPIRE].

[23] E. Witten, Multitrace operators, boundary conditions and AdS/CFT correspondence, hep-th/0112258 [INSPIRE].

[24] H.W. Lin, J. Maldacena and Y. Zhao, Symmetries near the horizon, JHEP 08 (2019) 049 [arXiv: 1904.12820] [INSPIRE].

[25] J. Maldacena and X.-L. Qi, Eternal traversable wormhole, arXiv:1804.00491 [INSPIRE].

[26] J.M. Maldacena, Eternal black holes in anti-de Sitter, JHEP 04 (2003) 021 [hep-th/0106112] [INSPIRE].

[27] S. Hirano, Y. Lei and S. van Leuven, Information transfer and black hole evaporation via traversable BTZ wormholes, JHEP 09 (2019) 070 [arXiv:1906.10715] [INSPIRE].

[28] P. Gao and H. Liu, Regenesis and quantum traversable wormholes, JHEP 10 (2019) 048 [arXiv: 1810.01444] [INSPIRE].

[29] B. Freivogel et al., Lessons on eternal traversable wormholes in AdS, JHEP 07 (2019) 122 [arXiv: 1903.05732] [INSPIRE].

[30] S. Gao and R.M. Wald, Theorems on gravitational time delay and related issues, Class. Quant. Grav. 17 (2000) 4999 [gr-qc/0007021] [INSPIRE].

[31] W.R. Kelly and A.C. Wall, Holographic proof of the averaged null energy condition, Phys. Rev. D 90 (2014) 106003 [Erratum ibid. D 91 (2015) 069902] [arXiv:1408.3566] [INSPIRE].

[32] M. Franz and M. Rozali, Mimicking black hole event horizons in atomic and solid-state systems, Nature Rev. Mater. 3 (2018) 491 [arXiv:1808.00541] [INSPIRE]. 